Proyecciones

Vol. 22, No 2, pp. 117-125, August 2003.

Universidad Católica del Norte

Antofagasta - Chile

\title{
A COMMUTATOR RIGIDITY FOR FUNCTION GROUPS AND TORELLI'S THEOREM *
}

\author{
RUBÉN HIDALGO \\ Universidad Técnica Federico Santa María, Valparaíso - Chile
}

\begin{abstract}
We show that a non-elementary finitely generated torsion-free function group is uniquely determined by its commutator subgroup. In this way, we obtain a generalization of the results obtained in [2], [3] and [8]. This is well related to Torelli's theorem for closed Riemann surfaces. For a general non-elementary torsion-free Kleinian group the above rigidity property still unknown.
\end{abstract}

2000 Mathematics Subject Classification. Primary $30 F 40$.

Key words and phrases. Kleinian groups, Function groups, Torelli's theorem, Hyperbolic 3-manifolds.

${ }^{*}$ This work was partially supported by projects UTFSM 12.03 .21 and Fondecyt $1030252,1030373$. 


\section{Introduction}

Let us assume that $S$ is a closed Riemann surface of genus $g \geq 2$. Associated to $S$ we have its homology group with integer coefficients $\mathrm{H}_{1}(S, \mathbf{Z}) \cong \oplus^{2 g} \mathbf{Z}$ and its complex vector space of holomorphic 1-forms $\mathrm{H}^{1,0}(S) \cong \mathbf{C}^{g}$. Using integration of 1 -forms over cycles we may see $\mathrm{H}_{1}(S, \mathbf{Z})$ inside $\mathrm{H}^{1,0}(S)^{*}$, the dual space of $\mathrm{H}^{1,0}(S)$, as a lattice. The quotient $J(S)=\mathrm{H}^{1,0}(S)^{*} / \mathrm{H}_{1}(S, \mathbf{Z})$ is called the Jacobian of $S$, which is a complex torus of dimension $g$ admitting a principal polarization given by the intersection form. Torelli's theorem asserts that $S$ is uniquely determined by its jacobian and its polarization. In fact, generically $S$ is only determined by its jacobian alone. Every known proof of Torelli's theorem uses the polarization to determine $S$.

Given any point $p \in S$, we have a conformal embedding $j_{p}: S \hookrightarrow J(S)$, defined by $j_{p}(q)=\left[\int_{p_{0}}^{q}\right]$. A discussion of this can be found, for instance, in [1]. We can lift $j_{p}(S)$ by the universal covering $\pi: \mathrm{H}^{1,0}(S)^{*} \rightarrow J(S)$ to obtain a Riemann surface $\widetilde{S}$, called the homology cover of $S$, so that $S$ is conformally equivalent to $j_{p}(S)=\widetilde{S} / \mathrm{H}_{1}(S, \mathbf{Z})$.

Let $F$ be a co-compact fuchsian group, acting on the hyperbolic plane $\mathbf{H}$, so that $S=\mathbf{H} / F$. We have that $\widetilde{S}=\mathbf{H} /[F, F]$, where $[F, F]$ is the commutator subgroup of $F$, that is, the subgroup of $F$ generated by all its commutators. In particular, if we forget the polarization of $J(S)$, then we may say that Torelli's theorem is essentially "equivalent" to the following.

Theorem 1 [8]. Let $F$ be a torsion-free co-compact fuchsian group. Then $F$ is uniquely determined by its commutator subgroup $[F, F]$.

In [2], [3] we have generalized the above result to certain types of nonelementary finitely generated torsion-free Kleinian groups (Schottky type groups and B-groups). In these notes, we prove that this still holds for finitely generated torsion-free function groups (Kleinian groups with an invariant connected component). The case of torsion-free reversing Fuchsian groups of the first kind has been studied in [6] and [7].

Theorem 2. Let $G$ be a finitely generated non-elementary torsion free function group. Then $G$ is uniquely determined by its commutator subgroup $[G, G]$.

The arguments in the proof still essentially the same as the one done in the previous notes, but the difference has to do with the correct the choice of the homology and cohomology, which is not natural, in this more general situation. This is the main reason for writing this note. 


\section{Remarks.}

(1) In higher dimensions the above property fails as can be seen from an example given in [3].

(2) If the group has no invariant components, then we have shown in [4] and [5] that for certain type of special Kleinian groups the above rigidity property still valid.

Let us recall that the homology cover of a manifold $M$ is the one defined by the commutator subgroup of the fundamental group of $M$. In this way, theorem 2 can be written in the language of 3-manifolds as follows.

Theorem 3. Let $M$ be a hyperbolic-three manifold with a non-abelian fundamental group $\pi_{1}(M)$. Assume that $M$ has a (conformal) boundary component $S$ (induced by its hyperbolic structure) such that the natural inclusion $i: S \hookrightarrow M$ induces a surjective homomorphism $i_{*}: \pi_{1}(S) \rightarrow$ $\pi_{1}(M)$. Then the hyperbolic structure of $M$ is uniquely determined, up to isometries, by the hyperbolic structure of its homological cover.

Proof. Let $G \cong \pi_{1}(M)$ be a uniformizing group of the manifold $M$ (of isometries of the hyperbolic three space) satisfying the hypothesis of the theorem. The group $G$ is necessarily a function group as a consequence of the surjectivity of $i_{*}: \pi_{1}(S) \rightarrow \pi_{1}(M)$. The result now follows from theorem 2 .

The above results can be seen as a step into the search of a Torelli's type theorem for analytically finite Riemann surfaces and stable Rieman surfaces.

\section{Some Basic Tools}

To prove theorem 2 we need some basic facts from Riemann surfaces theory. First, we need the following, which is an easy consequence of proposition E.10 in chapter $\mathrm{V}$ of [9].

Lemma 1. Let $G$ be a group of Möbius transformations and $K \neq\{I\}$ be a subgroup of $G$.

(1) If $K$ is a normal subgroup of $G$ and any of them is a non-elementary Kleinian group, then the other is also a Kleinian group sharing the same region of discontinuity. 
(2) If $K$ is a finite index subgroup of $G$ and any of them is a Kleinian group, then the other is also a Kleinian group with the same region of discontinuity.

Let now $T$ be an analytically finite Riemann surface, of signature $(g, k)$ and let $\bar{T}$ be its compactified Riemann surface of genus $g$. Set $H(T)$ to be the complex vector space generated by the holomorphic and antiholomorphic 1-forms on $\bar{T}$ and the holomorphic 1-forms on $T$ with at most simple poles at the punctures of $T$. The following is well known and can be found, for instance, in [1].

Lemma 2. The dimension of $H(T)$ is given by

$$
\operatorname{dim}_{\mathbf{C}} H(T)= \begin{cases}2 g, & \text { if } k=0 \\ 2 g+k-1, & \text { otherwise. }\end{cases}
$$

The pairing given by

$$
(w, \alpha) \in H(T) \times H_{1}(T, \mathbf{C}) \rightarrow \int_{\alpha} w \in \mathbf{C}
$$

defines a duality between $H(T)$ and $H_{1}(T, \mathbf{C})$.

To write the third observation we need some facts. Let $(G, \Delta)$ be a finitely generated torsion free non-elementary function group with commutator subgroup $K$. Assume we have a finite index subgroup $I<G$ containing $K$. We have then a finite sequence of holomorphic coverings

$$
\begin{gathered}
r: \Delta \rightarrow \Delta / K \\
t: \Delta / K \rightarrow \Delta / I \\
p: \Delta / I \rightarrow \Delta / G .
\end{gathered}
$$

We also have the respective coverings at the level of 3-dimensional manifolds (with boundaries):

$$
\begin{gathered}
R: \widehat{\Delta} \rightarrow \widehat{\Delta} / K ; \\
T: \widehat{\Delta} / K \rightarrow \widehat{\Delta} / I ; \\
P: \widehat{\Delta} / I \rightarrow \widehat{\Delta} / G,
\end{gathered}
$$

where $\widehat{\Delta}=\Delta \cup \mathbf{H}^{3}$.

We may now look at the induced homomorphism at the level of first homology with complex coefficients: $H_{1}(P): H_{1}(\widehat{\Delta} / I, \mathbf{C}) \rightarrow H_{1}(\widehat{\Delta} / G, \mathbf{C})$. 
Since the index of $I$ in $G$ is finite, the covering $P: \widehat{\Delta} / I \rightarrow \widehat{\Delta} / G$ has finite degree and, in particular, $H_{1}(P)$ is surjective homomorphism. Moreover, we know that the kernel of these homomorphism is generated by those loops in $\widehat{\Delta} / I$ which lift to loops on $\widehat{\Delta} / K$.

We have the natural inclusions

$$
\begin{gathered}
i_{\Delta}: \Delta \hookrightarrow \widehat{\Delta} ; \\
i_{K}: \Delta / K \hookrightarrow \widehat{\Delta} / K ; \\
i_{I}: \Delta / I \hookrightarrow \widehat{\Delta} / I ; \\
i_{G}: \Delta / G \hookrightarrow \widehat{\Delta} / G .
\end{gathered}
$$

The induced homomorphisms at the level of homology with complex coefficients are surjective. Let us denote the respective kernels by $N_{\Delta}=$ $H_{1}(\Delta, \mathbf{C}), N_{K}=\operatorname{ker} H_{1}\left(i_{K}\right)<H_{1}(\Delta / K, \mathbf{C}), N_{I}=\operatorname{ker} H_{1}\left(i_{I}\right)<H_{1}(\Delta / I, \mathbf{C})$ and $N_{G}=\operatorname{kerH}_{1}\left(i_{G}\right)<H_{1}(\Delta / G, \mathbf{C})$.

We have that $N_{G}$ is given by the homology classes of those loops in $\Delta / G$ which lift to loops on $\Delta / K$, and that $H_{1}(r)\left(N_{\Delta}\right)<N_{K}, H_{1}(t)\left(N_{K}\right)<N_{I}$, $H_{1}(p)\left(N_{I}\right)<N_{G}$.

Let us consider the induced surjective homomorphism on the quotient

$$
H_{1}(p): H_{1}(\Delta / I, \mathbf{C}) / N_{I} \rightarrow H_{1}(\Delta / G, \mathbf{C}) / N_{G},
$$

which is the same as

$$
H_{1}(P): H_{1}(\widehat{\Delta} / I, \mathbf{C}) \rightarrow H_{1}(\widehat{\Delta} / G, \mathbf{C}),
$$

whose kernel is generated by those loops on $\Delta / I$ that lift to loops on $\Delta / K$. If we denote by $N_{I}^{\perp}$ (respectively, $N_{G}^{\perp}$ ) the subspace of $H(\Delta / I)$ (respectively, $H(\Delta / G)$ ) orthogonal to $N_{I}$ (respectively, $N_{G}$ ), then the map

$$
p^{*}: N_{G}^{\perp} \rightarrow N_{I}^{\perp},
$$

defined by pull-back forms, is dual to the above surjective homomorphism and, in particular, it is injective. The image $P^{*}\left(N_{G}^{\perp}\right)$ corresponds to those forms in $N_{I}^{\perp}$ which are invariant under the action of pull-back of each transformation in the abelian group $G / I$. The following is just consequence of all the above observations.

Lemma 3. We have that

$$
\operatorname{dim}_{\mathbf{C}} N_{G}^{\perp}=\operatorname{dim}_{\mathbf{C}} H_{1}(\widehat{\Delta} / G, \mathbf{C})=\operatorname{dim}_{\mathbf{C}} H(\Delta / G)-l_{G},
$$

where $l_{G}$ denotes the maximal number of homologically independent simple loops in $\Delta / G$ which lift to loops on $\Delta / K$. 


\section{Proof of Theorem 2}

Let us consider two non-elementary finitely generated torsion free function groups $\left(G_{1}, \Delta_{1}\right)$ and $\left(G_{2}, \Delta_{2}\right)$ such that $\left[G_{1}, G_{1}\right]=\left[G_{2}, G_{2}\right]=K$. We want to prove that $G_{1}=G_{2}$. Set $I=G_{1} \cap G_{2}$ and $J=\left\langle G_{1}, G_{2}\right\rangle$.

Since $K$ is non-elementary and normal in both $G_{1}$ and $G_{2}$, we have from lemma1 that the region of discontinuity of the three of them is the same. Let us denote such a region by $\Omega$. Similarly, since $J$ is a subgroup of the normalizer of $K$, we have necessarily that $J$ is a Kleinian group with $\Omega$ as region of discontinuity.

Ahlfors' finiteness theorem asserts that $\Omega / G_{1}$ and $\Omega / G_{2}$ are each one a finite union of finite area Riemann surfaces. It follows that the (possible branched) covering $\Omega / G_{i} \rightarrow \Omega / J$ has finite degree, that is, both $G_{1}$ and $G_{2}$ have finite index in $J$ and, in particular, we have that $I$ has finite index in $G_{1}, G_{2}$ and $J$. It also follows from lemma 1 that $I$ has $\Omega$ as region of discontinuity.

Claim. There is a common invariant component $\Delta$ for $G_{1}$ and $G_{2}$.

Proof. If $\Delta_{1}=\Delta_{2}$, then we consider $\Delta=\Delta_{1}$. Assume that $\Delta_{1} \neq \Delta_{2}$. In this case, $I$ keeps invariant both components $\Delta_{1}$ and $\Delta_{2}$. Since $I$ has finite index in $G_{i}$, we have that $I$ is finitely generated. It follows from [10] that $I$ is then a quasifuchsian group and, as a consequence, $\Omega=\Delta_{1} \cup \Delta_{2}$. As a consequence of this, we have that $\Delta_{1}$ and $\Delta_{2}$ are invariant under both $G_{1}$ and $G_{2}$. In particular, we may take $\Delta=\Delta_{1}$.

Let us consider the following notation: $\Delta / I=R, \Delta / G_{1}=S_{1}, \Delta / G_{2}=$ $S_{2}, \Delta / J=X$. We denote by $t: \Delta \rightarrow \Delta / K, r: \Delta / K \rightarrow R$ and $p_{i}: R \rightarrow S_{i}$, $i=1,2$, the natural holomorphic coverings induced by the groups $K, I / K$ and $G_{i} / I$, respectively. Let us consider the following commutative diagram: 


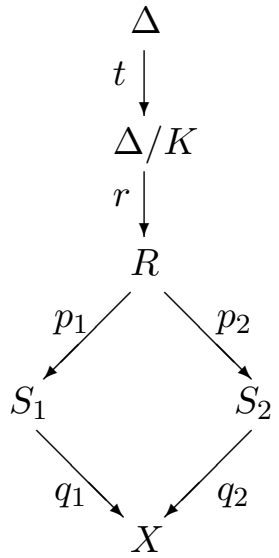

We have that the induced surjective homomorphisms

$$
H_{1}\left(p_{1}\right): H_{1}(R, \mathbf{C}) / N_{I} \rightarrow H_{1}\left(S_{1}, \mathbf{C}\right) / N_{G_{1}}
$$

and

$$
H_{1}\left(p_{2}\right): H_{1}(R, \mathbf{C}) / N_{I} \rightarrow H_{1}\left(S_{2}, \mathbf{C}\right) / N_{G_{2}}
$$

have the same kernel: the subspace generated by the classes of those loops on $R$ which lift to loops on $\Delta / K$. It follows that the forms in $N_{I}^{\perp}$ invariant under $G_{1} / I$ are the same ones invariant under $G_{2} / I$. In particular, these are the same which are invariants under the group generated by $G_{1} / I$ and $G_{2} / I$. It follows that the dimensions of these three spaces are the same.

By lemma 3, the dimension of the forms invariant under $G_{j} / I$ (respectively, the group generated by $G_{1} / I$ and $\left.G_{2} / I\right)$ is equal to $\operatorname{dim} H\left(S_{j}\right)-l_{j}$ (respectively, $\operatorname{dim} H(X)-l$ ), where $l_{j}$ (respectively, $l$ ) denotes the maximal number of homologically independent simple loops on $S_{j}$ that lift to loops on $\Delta / K$ (respectively, simple loops in $X$ for which a power lift to loops on $\Delta / K)$.

In particular, we have the equalities

$$
\text { (*) } \quad \operatorname{dim}_{\mathbf{C}} H\left(S_{j}\right)-l_{j}=\operatorname{dim}_{\mathbf{C}} H(X)-l .
$$

Let us denote by $g_{j}$ (respectively, $g$ ) the genus of $S_{j}$ (respectively, $X$ ) and by $k_{j}$ (respectively, $k$ ) the number of punctures of $S_{j}$ (respectively, $X$ ). We have necessarily that

(1) $g \leq g_{i}$ 
(2) $k \leq k_{i}$

(3) $g-l \leq g_{i}-l_{i}$

Inequalities (1) and (2) are clear. To obtain inequality (3), one consider a maximal set $\Sigma$ of pairwise disjoint homologically independent simple loops on $X$ all of them lifting to loops on $\Delta / K$.

Let $\widetilde{\Sigma}_{i}$ the lifting of $\Sigma$ to $S_{i}$. We have that $\widetilde{\Sigma}_{i}$ is a collection of pairwise disjoint simple loops that lift to loops on $\Delta$. We proceed to cut both $X$ and $S_{i}$ along those loops. Now glue discs along the new boundaries. We still having a (maybe branched) covering. The genus of the new surface obtained in this way from $X$ is $g-l$. The sum of the genera from the new surfaces obtained from $S_{i}$ is at most $g_{i}-l_{i}$. Now inequality (3) follows from area comparison.

The inequalities (1), (2) and (3) together the equality $(*)$ gives the equalities

$$
g=g_{i}, \quad k=k_{i} \quad \text { and } \quad l=l_{i} .
$$

We obtain in this way that the index of $G_{j}$ in $J$ is one and, as a consequence, $G_{1}=G_{2}$ and we have proved theorem 2 .

\section{References}

[1] H. Farkas and I. Kra. Riemann surfaces. Graduate Texts in Mathematics, Springer-Verlag.

[2] R. Hidalgo. Homology coverings of Riemann surfaces, Tôhoku Math. J. 45 (1993), 499-503.

[3] R. Hidalgo, Kleinian groups with common commutator subgroup, Complex variables 28, pp. 121-133, (1995).

[4] R. Hidalgo. Noded Fuchsian groups, Complex Variables 36, pp. 45-66, (1998).

[5] R. Hidalgo. Noded function groups. Contemporary Mathematics. 240, pp. 209-222, (1999).

[6] R. Hidalgo. Homology covering of closed Klein surfaces. Revista Proyecciones 18, pp. 165-173, (1999). 
[7] R. Hidalgo. A note on the homology covering of analytically finite Klein surfaces. Complex variables 42, pp. 183-192, (2000).

[8] B. Maskit. The homology covering of a Riemann surface, Tôhoku Math. J. 38, pp. 561-562, (1986).

[9] B. Markit. Kleinian Groups Grundlehren der Mathematischen Wissenschaften, Vol. 287, Springer-Verlag, (1988).

[10] B. Maskit. On boundaries of Teichmüller spaces and on kleinian groups II, Ann. of Math. 91, pp. 607-639, (1970).

Received : March 2003.

\section{Rubén Hidalgo}

Departamento de Matemática

Universidad Técnica Federico Santa María

Casilla 110 - V

Valparaíso

Chile.

E-mail: rhidalgo@mat.utfsm.cl 